\title{
¿GRAMÁTICA IMPLÍCITA O GRAMÁTICA EXPLÍCITA EN ENSEÑANZA DE SEGUNDAS LENGUAS?: ESTUDIO DE CAMPO
}

\author{
IMPLICIT GRAMMAR OR EXPLICIT GRAMMAR \\ IN SECOND LANGUAGE ACQUISITION?: A CASE STUDY
}

Jorge MARTí CONTRERAS ${ }^{1}$

Universitat Jaume I

\section{RESUMEN:}

El presente artículo es el resultado de una investigación pilotada entre estudiantes universitarios que pretende observar qué método gramatical, explícito o implícito, es más eficaz en el aprendizaje y adquisición de una L2. El estudio de campo se ha realizado en dos grupos de estudiantes homogéneos y paralelos en el marco de la asignatura Español económico y empresarial. Para ello se han diseñado materiales idénticos en el contenido y con un enfoque por tareas, pero con un método de gramática distinto en cada uno de los grupos, de forma que pudiera llevarse a cabo una visión contrastiva de los resultados.

En el artículo se plantea un marco teórico, se describe cómo se han diseñado los materiales y cuáles son los resultados obtenidos. Finalmente, se contrastan los resultados que serán significativos para que los estudiantes pueden maximizar la adquisición de conocimiento a lo largo del proceso de aprendizaje-enseñanza. Gracias al trabajo de campo se evidencia que el uso de gramática implícita mejora exponencialmente el rendimiento académico del alumnado, no solo a corto plazo.

PALABRAS CLAVE: gramática implícita, gramática explícita, enfoque por tareas; gramática; adquisición de segundas lenguas.

\section{ABSTRACT:}

This paper is the result of research conducted in the classrooms at the University of Valencia. The aim of this study is to analyse which grammar is better suited to teach Second Language Acquisition: deductive or inductive. At the Faculty of Economics we offer a course on Business Spanish. A case study was conducted in this course to study the effectiveness of bringing the classroom materials designed for following a task-based approach. The class was split into two groups in which in one, the grammar was deductive, and in the other, was inductive.

This study lays out a theoretical approach, describes how the materials are designed and what results were obtained. We also compare the results that are meaningful to maximise the acquisition of the knowledge during the learning-teaching process.

KEY WORDS: implicit grammar, explicit grammar, task-based approach grammar; Second Language Acquisition.

${ }^{1}$ Este trabajo se enmarca en el proyecto La atenuación pragmática en el español hablado: su variación diafásica y diatópico (FFI2013-40905-P), financiado por el Ministerio de Economía y Competitividad. 


\section{INTRODUCCIÓN}

El presente artículo es el resultado de una investigación llevada a cabo con alumnos de Español como Lengua Extranjera (E/LE) dentro del ámbito universitario. El estudio tenía como objetivo la verificación de nuestra hipótesis de trabajo: examinar qué tipo de gramática (implícita o explícita) es asimilada, a largo plazo, mejor por los estudiantes.

Numerosos investigadores se han dedicado al estudio de la gramática en la docencia de L2 (segundas lenguas). De manera general, el posicionamiento ante las diferentes propuestas es acometido desde una perspectiva ecléctica sin decantarse hacia ningún método o enfoque, y sin dar respuesta a cuál es la mejor manera de integrar la gramática en el proceso de aprendizaje. Así lo ilustran las palabras de Ortega Olivares (1998: 138), la cursiva es nuestra:

Las investigaciones aunque sugerentes y representativas de por dónde van las cosas, no dan respuestas, sin embargo, al cúmulo de preguntas que se plantea la integración de la enseñanza de la gramática en el currículo comunicativo; cómo influye la atención en la forma en la adquisición de una lengua, qué tipo de instrucción formal es más efectivo, cuál es el mejor momento para aplicar la técnica, qué rasgos lingüisticos pueden suministrarse con ella, o qué variables individuales del aprendiz refuerzan o no sus efectos. El trabajo por realizar es ingente. Una cosa sí parece cierta, sin embargo; la necesidad de promover el conocimiento consciente gramatical de los aprendices es ineludible, $\mathrm{y}$, debido a esto, la enseñanza de la gramática, sean cuales fueren las tendencias metodológicas que se desarrollan en el siglo que viene, deberán tenerla en cuenta o, mejor, considerarla como ingrediente esencial.

De la cita anterior se desprende la necesidad de realizar una investigación que demuestre qué tipo de enfoque gramatical se debería adoptar para maximizar el proceso de aprendizaje / adquisición de la lengua, siempre teniendo en cuenta los problemas. Autores como Llopis García, Real Espinosa y Ruiz Campillo (2012: 10) afirman que el problema en la didáctica de L2 no es la gramática, sino la visión que se tiene, y se ofrece de ella. Por su parte, Ortega Olivares (1998: 18) y Brucart (1997: 2) señalan dos problemas principales vinculados a la gramática; por un lado, los alumnos pueden aprender, por ejemplo, la formación de todos los tiempos verbales, y sin embargo, se encuentran con muchas dificultades a la hora de utilizarlos en un contexto real, fuera de las actividades proporcionadas en manuales de ejercicios gramaticales. Por otro lado, la gramática expuesta en el libro no se adapta a la realidad de los usuarios nativos, por ejemplo, en los manuales de clase se suele enseñar que el imperativo de venir para la segunda persona del singular es ven, pero no se suele explicar que si ya se ha insistido varias veces en la acción que se tenía que realizar, también puede emplear que vengas. Así pues, se reconoce que es imposible dar cuenta de todos los usos posibles de la gramática, y a la vez se reivindica no solo el aprendizaje gramatical, sino también el uso en el contexto adecuado. Tanto Brucart (1997: 18) como Ruiz Campillo (2007: 2) señalan que: 
Ser hablante competente de una lengua implica no solo dominar aisladamente su léxico y sus estructuras gramaticales, sino también saberlos articular en un discurso polifónico y saberlos utilizar de manera apropiada a los fines que el hablante intenta conseguir y al contexto social en que aquel se produce (Brucart, 1997: 18).

Es frecuente encontrar en el aula de segundas lenguas, que los aprendices estudien normas gramaticales y que ante un ejercicio gramatical al uso, planteado en un libro de texto, obtengan buenos resultados, pero que sin embargo, en el momento de ponerlos en práctica en un contexto real oral, se sientan incompetentes, e incluso no puedan mantener una interacción fluida. Por este motivo, para minimizar el impacto que supone trabajar varias habilidades al mismo tiempo, se han de desarrollar actividades comunicativas en las que sea necesaria la interacción.

A pesar de alcanzar niveles excelentes en lo que a fluidez respecta [...] no consiguieron niveles aceptables de corrección ni un uso completo de las funciones de la L2 (Alonso Raya y Martínez Gila, 1998: 326).

Tal y como se puede apreciar, son múltiples las dificultades planteadas a la hora de diseñar el sílabo de un curso y llevarlo al aula, muy en especial en lo referente a la efectividad en la asimilación gramatical. Con el objetivo de aportar un poco de luz en este aspecto, nos propusimos llevar a cabo un estudio de campo desde una perspectiva contrastiva. Se examina la evolución y los resultados en el ámbito gramatical de dos grupos de estudiantes que reciben una formación de español para fines específicos en la la asignatura Español Económico y Empresarial que se imparte en la Facultat de Economia, de la Universitat de València. Ambos grupos comparten los mismos objetivos, contenidos curriculares y la aproximación didáctica que sigue es el Enfoque Comunicativo Mediante Tareas (ECMT); el contraste solo se realiza en la explotación de la gramática: uno de los grupos recibe la gramática de forma inductiva (Coronado González, 1998: 85; Ellis, 2003: 165; Ambjoern, 2009: 17), y el otro de forma deductiva (Gómez del Estal y Zanón, 1996; Rodríguez Abella y Valero Gisbert, 1998; Gutiérrez Araus, 1998; Coronado González, 1999: 85).

\section{MARCO TEÓRICO}

El conflicto entre el conocimiento teórico de la gramática y su uso no es solo un hecho esporádico, sino más bien un problema frecuente en la enseñanza de L2, incluso en alumnos que estudian en programas de inmersión, tal y como afirman Alonso y Martínez (1998: 325):

Después de dos décadas, los resultados obtenidos por alumnos en contexto de inmersión, en los que cuentan con numerosas oportunidades de interacción y con un rico caudal de datos de la L2, no llegan a ser todo lo satisfactorio que cabría esperar. 
Nos encontramos, aún más, ante un conflicto que va más allá del aprendizaje en inmersión y del contexto, el de la concienciación gramatical. Para favorecer la adquisición de la gramática, los alumnos deben estar expuestos a un input estructurado, y el pertenecer a un programa de inmersión no está ligado con la organización del input sino con el caudal lingüístico al que se está expuesto. Cuando se habla de la concienciación gramatical deben ser tenidas en cuenta las necesidades específicas de cada alumno (o grupo de estudiantes), así como su contexto de aprendizaje. De acuerdo con VanPatten (1996) y Alonso Raya (2010: 19) las siguientes recomendaciones pueden ayudar al alumnado a obtener un mejor resultado en su proceso de aprendizaje ${ }^{2}$ :

- Enseñar solo un contenido cada vez.

- Mantener el significado de la forma objeto de estudio siempre presente.

- Procurar que los aprendices tengan que hacer algo con el input: mostrar acuerdo o desacuerdo, seleccionar entre alternativa, tachar lo que no es válido. De esta forma, pueden demostrar que comprenden.

- Usar input oral y escrito.

- Establecer secuencias que vayan de la frase al discurso.

- Mantener las estrategias de procesamiento psicolingüístico en mente.

- La forma / función gramatical objeto de atención debe ser la responsable de la comprensión del input.

- La actividad debe poner de manifiesto la operatividad de la regla o forma objeto de atención.

- Las actividades han de tener una finalidad y una contextualización claras y deben usar muestras de lengua verosímiles.

Todas las consideraciones señaladas por VanPatten (1996) y Alonso Raya (2010) se han tenido en cuenta a la hora de diseñar el material didáctico que se ha creado $a d$ hoc para el curso, como se verá en el próximo apartado se establecieron dos grupos para realizar un estudio contrastivo de campo. Gracias al ECMT cada tarea posibilitadora se trabaja con actividades contextualizadas y verosímiles dentro del área del español económico y empresarial; se procura desarrollar solo un aspecto gramatical que está siempre presente en la actividad; a lo largo de la unidad los alumnos son expuestos a input escrito, oral o audiovisual, y se les pide realizar actividades en las que sea esencial la comprensión y el uso de la norma estudiada.

Por otro lado, si se tiene en cuenta, además de la estructuración del input, la integración de la gramática en el contexto de aprendizaje, resulta idóneo el uso del ECMT ya que, en primer lugar, contextualiza las actividades e integra conocimientos gramaticales, y en segundo lugar, propone la resolución de tareas (proyectos o actividades) en donde la interacción (oral o escrita) es esencial. En los albores del Enfoque comunicativo estaban completamente disociados comunicación y gramática, ya que se esperaba que el alumno fuera capaz de aprender de forma autónoma la gramática sin necesidad de estudiarla, pero «hoy en día, se ha reconsiderado el papel de la gramática y se están llevando a cabo intentos de reconciliarla con el enfoque

${ }^{2}$ Las seis primeras recomendaciones son de Alonso Raya (2010: 19), el resto de VanPatten (1996). 
comunicativo, aunque la cuestión continúa siendo problemática» (Llopis García, 2009: 5). Es precisamente en estos «intentos» en los que se basa el presente artículo.

Se han diseñado materiales didácticos ad hoc, que han sido experimentados con dos grupos de alumnos para comprobar, o refutar, las hipótesis de trabajo adoptadas, e intentar resolver el reto planteado por Coronado González (1998: 91) y Baralo (2009: 17) en el que invitaban a filólogos, pedagogos y profesores de $\mathrm{E} / \mathrm{LE}$ a indagar cómo enseñar la gramática. Se cuenta con aportaciones previas de Zanón et al. (1990), así como con el reto expresado por Castañeda Castro (1988: 67), quien propone que no solo se integre la gramática en el sílabo, sino también la integración, de forma coherente, de la gramática dentro del enfoque comunicativo por tareas.

\subsection{Aportaciones teóricas a la gramática}

A lo largo de este apartado se presentan y discuten las principales aportaciones teóricas sobre la gramática en la enseñanza de L2 y el modo de integrarlas en el enfoque comunicativo. Para acercarnos a este punto, se partirá de la reflexión planteada por Ortega Olivares (1998: 138), y que ya se vio al inicio de este artículo.

Ortega denuncia una serie de problemas relativos al trabajo de la gramática en E/LE, y muestra el hecho de que todavía no se ha llegado a una buena solución sobre cómo integrarla en la programación. Trabajar la gramática en el proceso de adquisición / aprendizaje de L2 supone tener en cuenta dos ejes: el primero de ellos tiene que ver con la forma en la que el docente presenta la gramática en el aula: implícita o explícita; el segundo eje atiende a la manera en que el alumno la adquiere: inductiva o deductiva, en este artículo tendremos en cuenta solo el primero de los ejes.

Cuando se hace referencia a enfoques en contraste, se deben cruzar los cuatro tipos de enseñanza gramatical: deductiva, inductiva, explícita e implícita. Dependiendo de cómo se busque llevar la gramática al aula, quien diseñe un curso estará adoptando una metodología o enfoque u otro, tal y como se muestra en la tabla $1^{3}$ :

${ }^{3}$ La tabla es una adaptación de la información obtenida gracias a Leech (1989: 22), Rodríguez y Valero (1998: 434), Salazar (2006: 6) e Izquierdo (2008: 45). 


\begin{tabular}{|c|c|c|}
\hline & Deductiva & Inductiva \\
\hline$\stackrel{\frac{\pi}{2}}{\frac{\pi}{2}}$ & $\begin{array}{l}\text { a) Método tradicional } \\
\text { de gramática-traducción } \\
\text { Modelos teóricos-formales }\end{array}$ & $\begin{array}{l}\text { c) Gramática tradicional } \\
\text { Metodología Natural o Directa } \\
\text { El estudiante tras una primera exposición a } \\
\text { un input apropiado, hace una hipótesis y } \\
\text { extrae una primera regla provisional }\end{array}$ \\
\hline 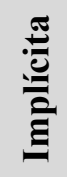 & $\begin{array}{c}\text { b) Método audiolingual } \\
\text { Ejercicios o prácticas gramaticales } \\
\text { (ejercicios de repetición, de huecos } \\
\text { y estructurales) }\end{array}$ & $\begin{array}{l}\text { d) Enfoque comunicativo } \\
\text { Muestra de lengua real }\end{array}$ \\
\hline
\end{tabular}

Tabla 1. Tipos de metodología en enseñanza de gramática

Cuando un estudiante adquiere la gramática, habitualmente no sigue solo uno de estos métodos, sino que la manera de procesarla puede ser desde perspectivas diferentes ${ }^{4}$, tal y como muestra la Tabla 1. Tipos de metodología en enseñanza de gramática a partir de las combinaciones posibles de los cuatro tipos. Sobre las características de cada una de las combinaciones resultantes, se puede consultar abundante literatura, entre la que destaca Fuentes de la Rosa (1992: 112). Veamos con detalle a continuación cada uno de estos métodos.

\subsection{La gramática explícita}

El primero de los dos ejes relativos a la enseñanza de la gramática (implícita o explícita ${ }^{5}$ ) atiende a la forma en que el profesor presenta la gramática en el aula. El aprendizaje explícito se basa en la exposición de las reglas a los alumnos por parte del profesor, se busca así que la gramática se adquiera conscientemente (Ellis, 1994: 643; Rodríguez Abella y Valero Gisbert, 1998: 434; Gutiérrez Araus, 1998: 2, Widodo, 2006: 125; Ambjoern, 2009: 5). Las características del aprendizaje explícito de la gramática, según Gómez del Estal (2004: 89) son:

- El aprendizaje está formado por reglas o representaciones abstractas del funcionamiento gramatical.

- Los alumnos analizan las reglas gramaticales, ya que pueden ser descritas y clasificadas.

- Es conocimiento explicativo, ya que las reglas dan cuenta objetivamente del funcionamiento del lenguaje utilizado para la comunicación.

- El alumno puede acceder y hablar sobre las reglas sin esfuerzo. Es lo que dice el alumno sobre la gramática de la lengua.

\footnotetext{
${ }^{4}$ Rodríguez Abella y Valero Gisbert (1998: 435) hablan de la «Adquisición [de la gramática de forma] indirecta, aunque no se descarta la reflexión lingüística deductiva».

5 Estas metodologías han sido estudiadas por autores como Gómez del Estal y Zanón (1996), Salazar (2006), Ruiz Campillo (2007), Aguado-Orea y Baralo (2007), Izquierdo (2008).
} 
La función básica de la gramática explícita es la monitorización (revisar y corregir lo que se ha producido). Para el proceso de monitorización el estudiante debe darse cuenta de que ha cometido un error, tiene que conocer la forma adecuada, y finalmente, ha de tener tiempo suficiente para corregir su producción.

La gramática explícita presenta algunas ventajas. Principalmente, se centra en el estudio directo del aspecto gramatical que el alumnado debe aprender y que está descrito en el sílabo del curso, por lo que permite ahorrar tiempo. Inmediatamente después de haber introducido la explicación gramatical se plantean actividades prácticas; en algunos casos pueden ser más simples y claramente explicadas a través de ejemplos. Se puede destacar también que si se trabaja con estudiantado adulto, y siguiendo simultáneamente un enfoque deductivo, se respeta la madurez del aprendiz, dado que se busca continuamente la reflexión sobre lo que se está aprendiendo y se reconoce el papel cognitivo del proceso de aprendizaje de lenguas vs. el producto. En otras palabras, se busca de manera parcial conseguir una autonomía en el aprendizaje.

Otra de sus ventajas es que colma las expectativas por defecto de la mayoría de estudiantes sobre el aprendizaje en clase, especialmente de aquellos que tienen un estilo analítico. Llopis García (2009: 3) añade que promueve el crecimiento de la interlengua y permite llegar a un grado de fluidez en las fases finales del aprendizaje. Asimismo, la autora, al referirse al estudiante en inmersión señala que ejercita y desarrolla su interlengua en un ambiente controlado que le ayudará a lograr precisión y corrección. $\mathrm{Si}$, por el contrario, no se encuentra en inmersión, afirma que el estudiante tendrá suficientes oportunidades, y la calidad necesaria para aprender la lengua extranjera con éxito. Por último, si se trabaja con fines comunicativos, el conocimiento explícito desempeña un papel de monitorización, y por lo tanto acelera el proceso de adquisición de rasgos como conocimiento implícito y permite llegar a un nivel más alto de competencia (Coronado González, 1998: 82-3).

La gramática explícita también plantea algunos inconvenientes (Gómez del Estal, 2004: 14; Widodo, 2006: 127-8). Por un lado, para algunos alumnos el aprendizaje de un idioma es simplemente conocer la gramática. La explicación gramatical rara vez es tan fácil de recordar como otras formas de presentación, como por ejemplo la demostración. Además, se necesita a un profesor al frente del aula que formule las explicaciones, lo cual también dificultará la participación y el grado de involucramiento del alumno de forma inmediata. Y, empezar la lección con presentación gramatical puede romper negativamente las expectativas de los aprendices, que pueden no ser capaces de comprender el metalenguaje, aunque si bien es cierto, gran parte de los estudiantes están acostumbrados a la gramática explícita y esto es lo que desean de los cursos, pero, se corre el riesgo de que sirva solo para la resolución de ejercicios a corto plazo, sin garantizar su interiorización y posterior uso. Por último, es muy importante reconocer que en ocasiones el conocimiento explícito de la gramática no comporta un uso pragmático adecuado (Bardovi-Harlig, 2003: 25). 


\subsection{La gramática implícita}

En cuanto a la gramática implícita, mediante este sistema el alumno no es consciente de que está adquiriendo normas de construcción gramatical, las aprende de forma intuitiva. Winter y Reber (1994: 117), Rodríguez Abella y Valero Gisbert (1998: 434), y, Aguado-Orea y Baralo (2007: 126) definen el aprendizaje implícito como la habilidad humana para derivar información acerca del mundo de un modo inconsciente, no reflexivo. Las características del conocimiento implícito de la gramática, señaladas por Gómez del Estal (2004: 10) son las siguientes:

- Se compone de unidades sin analizar de dos tipos: frases-fórmula y reglas intuitivas.

- Las frases-fórmula son producciones lingüísticas sin analizar, aprendidas en bloque, como ¿qué tal?, o frases con huecos que pueden ser ocupados por palabras funcionalmente equivalentes: ¿puedes pasarme...?

- Las reglas intuitivas forman la gramática que subyace cuando el alumno habla o escribe la lengua espontáneamente.

- El conocimiento de la lengua se manifiesta a través de juicios de gramaticalidad sobre muestras de lengua en los que el alumno especula sobre el funcionamiento del sistema.

- No es accesible conscientemente. Por ejemplo, los hablantes de español como lengua materna son incapaces, en su mayoría, de explicar el funcionamiento gramatical de sus producciones.

El conocimiento implícito es el utilizado en el enfoque audiolingual y en el enfoque comunicativo, como muestra la Tabla 1. Tipos de metodología en enseñanza de gramática. Nos interesa especialmente porque nuestro estudio se centra en el enfoque comunicativo y porque es el que hipotéticamente dará mejores resultados; de hecho, es la metodología empleada en el diseño del material didáctico de uno de los dos cursos de 15667 Español económico y empresarial.

El conocimiento implícito puede ser construido a partir de tres mecanismos, de acuerdo con Gómez del Estal (2004: 11):

1) A partir del análisis del intake 6 .

a. El alumno presta atención a un fragmento de input desconocido sobre el resto de caudal lingüístico.

b. Comparación $X-X$. Compara la regla que opera en el intake $\mathrm{X}$, con la regla ya interiorizada $X$.

c. Interiorización. Si las conclusiones del $\mathrm{X}-\mathrm{X}$ son consideradas suficientes para la coherencia del sistema lingüístico en construcción.

2) Mediante la reestructuración del sistema lingüístico LE (o interlengua) del alumno.

a. Atención a una estructura $X$ del intake.

b. Inferencia / formación de hipótesis.

\footnotetext{
${ }^{6}$ Input son las muestras de lenguas a las que tiene acceso un usuario, intake corresponde a la parte lingüística que ha podido asimilar, y, el output es la producción realizada por estudiante, tanto de forma oral como de forma escrita.
} 
c. Cotejo por acumulación de evidencia empírica, por producción en lengua extranjera y feedback, por la enseñanza explícita de la regla, o por la interacción en situaciones de comunicación con input y output comprensibles.

3) Desde el conocimiento explícito ${ }^{7}$. El conocimiento implícito y el explícito interactúan permanentemente.

En el primero de los mecanismos, el análisis del intake, el alumno es expuesto a un nuevo input para que observe cómo se ha construido el mensaje, y para que contraste la nueva información con la que ya había adquirido anteriormente; si es lógica, la norma creada pasará a formar parte de la interlengua del estudiante, y por lo tanto, será interiorizada. En el segundo caso, la reestructuración del sistema lingüístico del estudiante, el individuo parte de un conocimiento previo, y siendo también sometido a un nuevo caudal lingüístico, esta vez compara la nueva hipótesis con las inferencias extraídas con anterioridad y, si es necesario, modifica la norma que había creado en la interlengua, pero esta la utilizará para la creación y emisión de mensajes lingüísticos. De acuerdo con el último mecanismo, también se puede llegar al conocimiento implícito desde el conocimiento explícito, pero para que tenga lugar este trasvase de conocimiento va a ser necesario mucho esfuerzo, y por lo tanto, no es rentable.

Como se ha señalado anteriormente, el conocimiento implícito depende de cómo el profesor expone la gramática en el aula. Presentamos, como ejemplo, una cadena ${ }^{8}$ de actividades del material didáctico diseñada para el curso:

\section{Ejemplo núm. 1}

ACTIVIDAD 1 Aquí tienes dos correos electrónicos dirigidos a una persona que se llama Carmen, ¿en qué crees que trabaja Carmen?

\begin{tabular}{|c|c|}
\hline \multirow{2}{*}{$\begin{array}{l}\text { 2.19\% de } 1953.13 \text { MB usados } \\
-\square \text { jorge.marti@uv.es }\end{array}$} & (1) Asunto \\
\hline & Sin mensajes \\
\hline Bendelade entrada & Hola, Carmen: \\
\hline $\begin{array}{l}\text { Enviados } \\
\text {-7) Papelera } \\
\square \text { Sent } \\
\square \text { marcon2_paquetes }\end{array}$ & $\begin{array}{l}\text { Tú ya sabes que yo siempre digo que en el trabajo necesito limpieza. } \\
\text { Todos los días tengo problemas con mis compañeros. Pongo las } \\
\text { herramientas en el lugar adecuado, pero cuando salgo y traigo algo } \\
\text { nuevo que necesito, ya está fuera del lugar. }\end{array}$ \\
\hline & $\begin{array}{l}\text { Todavía no conozco quién es la persona que hace todo esto... hay veces } \\
\text { que parezco un poco tonto, porque no me doy cuenta de las cosas, o no } \\
\text { oigo, o no veo qué sucede. }\end{array}$ \\
\hline & ¡Ahora caigo! Creo que quieren gastarme una broma. \\
\hline & Te agradezco la atención prestada. \\
\hline
\end{tabular}

\footnotetext{
${ }^{7}$ Se estudiará más adelante, cuando hablemos de la hipótesis de Krashen

${ }^{8}$ Las actividades y las tareas siempre deben estar integradas en una secuencia de actividades y nunca de forma aislada.
} 


\begin{tabular}{|c|c|}
\hline \multirow{7}{*}{ 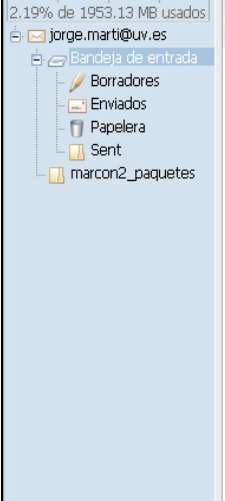 } & Sin mensajes \\
\hline & Carmen: \\
\hline & ¡Ya estoy harto y no importa lo que diga ese individuo! \\
\hline & $\begin{array}{l}\text { Quizás él tenga un poco de obsesión por el orden. No me gusta que él } \\
\text { ponga las condiciones que le salgan, perdona por la expresión, ni que } \\
\text { traiga al trabajo tonterías. }\end{array}$ \\
\hline & $\begin{array}{l}\text { Tal vez conozcas la última que ha hecho, y es posible que te parezca una } \\
\text { estupidez y seguramente oigas comentar a todos que no pasa nada... pero } \\
\text { es imposible soportar esta situación, ojalá se caiga y le tenga que irse a } \\
\text { casa una temporadita. }\end{array}$ \\
\hline & ¡Y que no me agradezca lo que he hecho siempre por él! \\
\hline & Lamento mucho esta situación, saludos, \\
\hline
\end{tabular}

ACTIVIDAD 2 Si te fijas, en ambos correos electrónicos hemos señalado en negrita algunos verbos ¿podrías compararlos? ¿Qué similitudes y diferencias encuentras?

ACTIVIDAD 3 Completa la tabla con los verbos en presente que aparecen en los correos electrónicos anteriores. Añade también el infinitivo.

\begin{tabular}{|c|c|c|}
\hline Infinitivo & $\begin{array}{c}\text { Presente de } \\
\text { indicativo }\end{array}$ & $\begin{array}{c}\text { Presente de } \\
\text { subjuntivo }\end{array}$ \\
\hline dener & & \\
\hline & & ponga \\
\hline & traigo & \\
\hline & palgan \\
\hline conocer & & \\
\hline & & oigas \\
\hline & agrezco & caiga \\
\hline caer & & \\
\hline & &
\end{tabular}

\begin{tabular}{|c|c|}
\hline \multicolumn{2}{|c|}{$\begin{array}{c}\text { Terminaciones } \\
\text { Pres. subj }\end{array}$} \\
\hline -AR & -ER/-IR \\
\hline -e & -a \\
\hline -es & -as \\
\hline -e & -a \\
\hline -emos & -amos \\
\hline -éis & -áis \\
\hline -en & -an \\
\hline
\end{tabular}

En esta secuencia de actividades el alumno va a seguir el primero de los mecanismos de adquisición (conocimiento implícito). Se presentan dos correos electrónicos, dirigidos a un mismo receptor, y en los que se narran hechos diferentes. Se destaca en distinta tipografía (letra negrita) el nuevo input lingüístico, que es reconocido por los alumnos, y a empiezan a comparar el intake con el input, en este caso, las formas gramaticales en presente de indicativo y las formas de presente de subjuntivo. En la actividad 2, se invita al estudiante a la reflexión para que extraiga una serie de conclusiones: la formación del presente de subjuntivo depende de la irregularidad en la primera persona singular del presente de indicativo; se emplea indicativo o subjuntivo para expresar distintas funciones comunicativas. Una vez comprobada la validez de esta hipótesis, es interiorizada.

Veamos los puntos fuertes de la enseñanza de la gramática de forma implícita en el proceso de aprendizaje. El alumno tiene un papel de receptor / emisor totalmente activo, frente a otros enfoques que conceden a los alumnos un papel pasivo; esta 
pasividad puede llevar a la desmotivación. Además, si se descubren las normas durante la realización de las tareas comunicativas se consiguen dos objetivos, por un lado las reglas gramaticales son adquiridas, y por otro, es una actividad extra para practicar la comunicación. Para llegar a este punto, los aprendices son entrenados para estar familiarizados con el descubrimiento de las normas, hecho que aumenta la autonomía en el aprendizaje y la autosuficiencia.

También se han señalado diversos inconvenientes en este método implícito. Así por ejemplo, se ha destacado el excesivo consumo de tiempo y energía por parte del alumno hasta que es capaz de llegar a la regla, ya que necesita más tiempo para establecer normas e integrarlas en la interlengua, y después, gracias al nuevo input, debe confirmar o refutar las hipótesis previas. El mismo inconveniente se puede aplicar al profesor en la elaboración del material, puesto que las unidades deben ser adaptadas a las necesidades específicas de los alumnos, y deben estar diseñadas gradualmente para que el alumno pueda llegar a las conclusiones, paso a paso, por lo que trabajar la gramática de esta forma supone más inversión de tiempo, que no siempre resulta rentable. Otro aspecto a tener en cuenta, es la posibilidad de que los conceptos transmitidos implícitamente puedan llevar a los alumnos a inducir la teoría equivocada de la norma enseñada. Por último, este modo de enseñar la gramática podría frustrar a alumnos con estilos de aprendizaje tradicional (o con experiencia de aprendizaje), quienes esperan que las reglas gramaticales vengan dadas por el profesor. Por estos motivos hay autores que no recomiendan que se estudie la gramática exclusivamente de forma implícita.

Existe una tercera posición en el modo de enseñanza de la gramática que destaca la hipótesis de la interfaz planteada por Baralo (Baralo, 1995: 65; 1996: 17), según la cual:

La posición de la interfaz sostiene que sí es posible que se produzca algún tipo de trasvase del conocimiento explícito al implícito, o no consciente, entendiendo ese movimiento como un continuo más que como dos sistemas discretos. Es decir, es factible que el aprendizaje se convierta en adquisición, el conocimiento explícito en implícito, los procesos controlados de la memoria a corto plazo en procesos automáticos de la memoria a largo plazo, dependiendo del tipo de ejercitación que se realice en la clase de E/L2. Esta transformación se puede conseguir a través del uso, de la práctica con necesidades comunicativas, de la interacción y de la negociación (Baralo, 1995: 65).

Mediante la formulación de esta hipótesis se defiende la posibilidad de trasvase de conocimiento explícito al implícito (tal y como queda reflejado en el Esquema 1. Conocimiento explícito y conocimiento implícito, se estudiará en detalle en las próximas páginas, cuando se traten los conceptos de adquisición y aprendizaje). Por ejemplo, si se presenta un paradigma verbal y se trabaja lo suficiente en un contexto real adecuado, no solo mediante actividades repetitivas y mecánicas sino también de reflexión, se podría realizar la transformación hacia el conocimiento implícito, en el que no es necesario que el alumno memorice las normas gramaticales, sino que las adquiera y sea capaz de usarlas adecuadamente. Por lo tanto, lo que se estaría haciendo realmente son 
actividades comunicativas, una vez que se ha aprendido la gramática de forma explícita, de esta manera se intenta que el estudiante logre fluidez a la hora de producir output.

Otro inconveniente sería que cada alumno tiene un estilo de aprendizaje diferente y el profesorado no se puede adaptar siempre al estilo de cada uno de ellos. No obstante, el alumno dispone de tiempo y técnicas suficientes de autoaprendizaje como para readaptar el nuevo input según su estilo de aprendizaje. Además, cuando se hace referencia a la necesidad de un cierto dominio previo de la lengua, se señala que el input ha de ser comprensible para que el discente sea capaz de comprender la nueva información sin necesidad de una explicación teórica previa, y para ello se necesitan grupos de estudiantes con el mismo conocimiento de lengua. Por otro lado, en la cita de Coronado González (1998: 85) de arriba, se hacía referencia a la falta de material en el mercado editorial; no obstante, la década transcurrida desde que se formularon estas palabras hasta ahora, ha sifo fructífera, y estamos asistiendo a un cambio sustancial en lo relativo a la producción de material didáctico basado en la inducción.

Autores como Sánchez Pérez (1982: 150), Castañeda Castro (1988: 68), Rodríguez Abella y Valero Gisbert (1998: 434) hablan de la comparación positiva del aprendizaje inductivo de un estudiante de E/LE y de un niño que aprende su lengua materna. Sin embargo, otros se postulan en contra de este punto de vista, pues el contexto y las condiciones de aprendizaje no son semejantes:

No admito, antes bien tengo por absurda la opinión de los que dicen: «Aprended un idioma extranjero como habéis aprendido vuestra lengua nativa». Y esto por muy varias razones. La primera, porque no hay paridad entre el niño inconsciente y el joven adulto que piensa y raciocina; la segunda porque el niño dedica todos los momentos de su vida a aprender y el adulto solo puede consagrar al estudio de la lengua que aprende algunos cuartos de hora. El niño no oye otras voces ni otros sonidos que los del idioma nativo, el adulto muy al contrario. [...] y es un absurdo pretender que el adulto aprenda bien un idioma extranjero por el mismo procedimiento que aprendió el de su madre (García Ayuso, 1879: Prólogo apud Viña, 2005: 192-3).

García Ayuso, hace más de un siglo, restaba importancia al aprendizaje de lenguas por inmersión, ya que reconocía que el proceso es totalmente diverso por el desarrollo cognitivo de los individuos y por la integración psicosocial de cada uno de ellos.

Tanto docente como discente desean enseñar/adquirir la L2 a partir de un enfoque o una metodología que sea lo más motivadora posible para incentivar el progreso. El progreso ha de seguir una lógica convencional para conseguir la retención a largo plazo. Esto es, a partir de un proceso inductivo.

\subsection{Del conocimiento aprendido al adquirido}

Los términos adquisición de la lengua y aprendizaje de la lengua (conceptos estudiados por Larsen-Freeman y Long (1994: 273), Baralo (1996: 7), Matte Bon (1998: 63), Ambjoern (2009: 5) han sido utilizados como sinónimos durante mucho tiempo -incluso hoy en día-, algunos autores recogen la definición de sendos términos, con 
matices distintivos (Baralo, 1995: 65; Brucart, 1997: 20; el Marco común europeo de referencia para las lenguas [MCER], 2002: 137; y Llopis García, 2009: 2):

Adquisición de la lengua: «Los conocimientos y las capacidades, no explícitamente enseñados, que permiten utilizar una lengua no nativa y que son el resultado de una exposición directa al texto o a una participación directa en situaciones comunicativas». Además «son procesos mentales automáticos, basados en la memoria a largo plazo, que suponen un conocimiento implícito de los elementos sustantivos y de las reglas del sistema lingüístico».

Aprendizaje de la lengua: «proceso por el cual se consigue la capacidad lingüística como resultado de un proceso planteado, sobre todo, mediante el estudio académico en un marco institucional», podemos añadir «está basado en la memoria a corto plazo, que supone un conocimiento explícito de los componentes y de las reglas de la lengua» (MCER, 2002: 137).

El aprendizaje, por tanto, se entiende como el proceso de estudio de una lengua para poder usar ciertos rasgos lingüísticos, que por regla general, son utilizados correctamente durante un periodo de tiempo relativamente corto; mientras que la adquisición respondería a la integración del uso comunicativo de la lengua de la L2 conseguida a través de la práctica en procesos comunicativos, para poder usar correctamente la lengua; el hablante debe haber realizado todo un proceso de concienciación y reflexión gramatical de forma implícita.

En el apartado anterior, se ha descrito la hipótesis de la interfaz, en la que se defiende el paso del conocimiento explícito al implícito (Baralo, 1995: 65), y por lo tanto, se pasaría del aprendizaje a la adquisición de la gramática. Esta hipótesis puede ser complementada con la hipótesis del input de Krashen (1985), que ha sido descrita por autores como Brucart (1997: 20), Baralo (1995: 63-68 y 2009: 7), Ortega Olivares (1998: 4) y Llopis García (2009: 2). Según esta hipótesis se debe diferenciar entre conocimiento adquirido y conocimiento aprendido. El primero corresponde al saber inconsciente que desemboca en el uso de la lengua, mientras que el segundo es consciente y se logra gracias a la instrucción explícita y capacidades cognitivas. En el aprendizaje de lenguas, el conocimiento se consigue a través de la memorización de reglas y principios de gramática. En ejercicios mecánicos el usuario puede tener éxito, pero no será así en actividades comunicativas naturales, ya que solo se puede realizar una producción fluida si se han adquirido los conocimientos.

A pesar de que el trasvase del conocimiento explícito al implícito es posible, autores como Baralo (1995) o Ortega Olivares (1998), destacan que es prácticamente insignificante y además se conseguiría solo a través de actividades comunicativas:

[...] sí es posible que se produzca algún tipo de trasvase del conocimiento explícito al implícito [...] esta transformación se puede conseguir a través del uso, de la práctica con necesidades comunicativas, de la interacción y de la negociación (Baralo, 1995: 65).

La función del conocimiento aprendido es secundaria, cuando no irrelevante, puesto que todo (o casi todo) se mueve a instancias del conocimiento adquirido: gracias a él emerge la producción espontáneamente y se activa la adquisición de la lengua meta en 
los mismos términos que la materna. Lo aprendido, en cambio, no está en la base de ningún desarrollo espontáneo [...]. Se trata, como se ve, del argumento tantas veces utilizado en contra de la enseñanza de la gramática (Ortega Olivares, 1998: 4).

La relación entre enseñanza y conocimiento gramatical de una lengua extranjera se puede resumir con esta imagen que ha sido adaptada a partir de la propuesta de varios autores (Brucart, 1997: 21; Ellis, 1993; Lee y VanPatten, 1995: 2; Gómez del Estal y Zanón, 1999: 77; Baralo, 2003: 31-43; Alonso, 2004: 2 y 2010: 10; Gómez del Estal, 2004: 11; Dekeyser, 2005: 315):

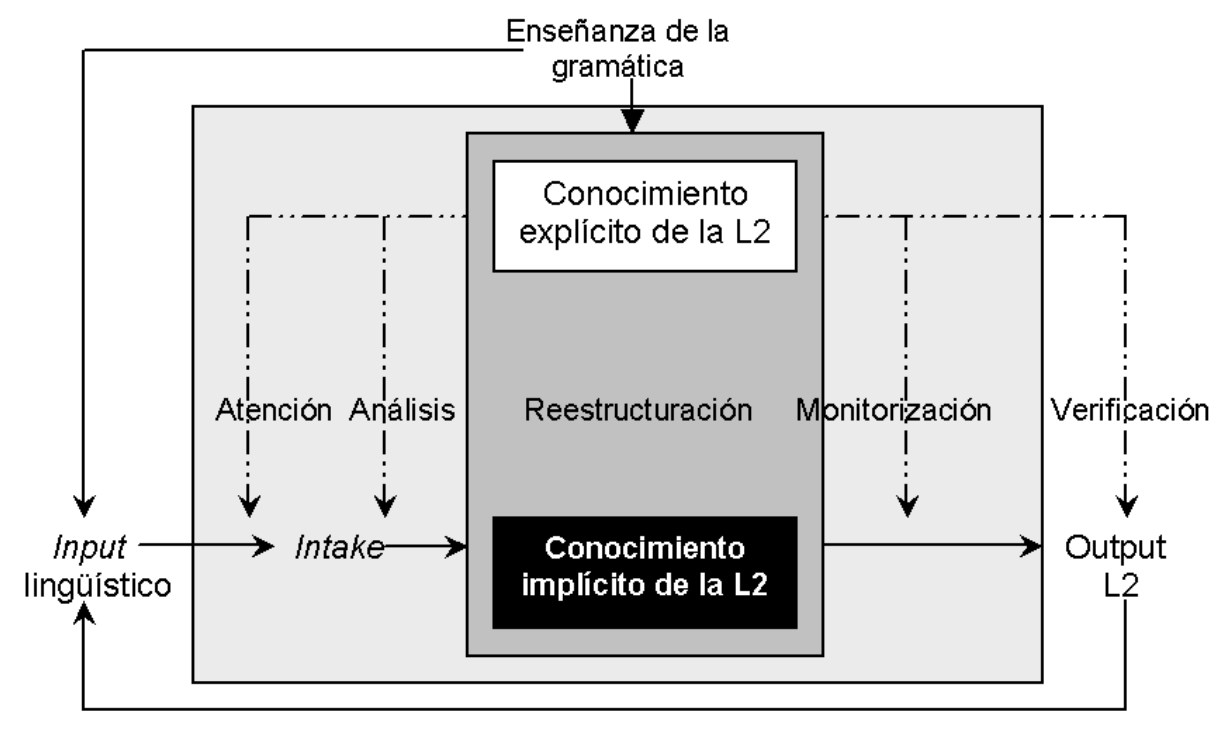

Esquema 1. Conocimiento explícito y conocimiento implícito

Se ha explicado que el conocimiento gramatical adquirido por un estudiante sigue el esquema que se acaba de presentar. El conocimiento puede ser implícito o explícito, si se opta por el aprendizaje de forma implícita, es expuesto a un input lingüístico (programas de radio o televisión, textos periodísticos, literarios... diálogos... ejercicios especialmente diseñados para la reflexión). El input provocará el intake, esto es, la atención directa del estudiante hacia el input, ya que un individuo no es capaz de asimilar toda la información a la que es expuesto. Llegados a este punto, el alumno habrá adquirido el conocimiento, y para finalizar el proceso tan solo le faltará el output, es decir, comprender y/o producir en L2.

En cambio, para aprender la gramática de forma explícita, al alumno se le facilitarán unas normas gramaticales, a las que prestará atención, analizará y reestructurará el conocimiento previo. El proceso de monitorización será el siguiente paso, en el que el estudiante mentalmente corregirá la producción (son las décimas de segundo en que, en ocasiones, el alumno calla antes de la producción; si necesitara mucho tiempo, se podría entorpecer el proceso de comunicación e incluso podría llegar a imposibilitarlo). Y finalmente, llegará la producción en L2. 
El esquema anterior (Esquema 1. Conocimiento explícito y conocimiento implícito) muestra cómo, en definitiva, el conocimiento explícito de la gramática y el implícito no son dos modos totalmente estancos, sino que la información aprendida del conocimiento explícito puede convertirse en contenidos adquiridos, tal y como encontramos en la hipótesis de la interfaz. Según Coronado González (1998: 82), conocer previamente el funcionamiento de un rasgo gramatical permite que el estudiante lo perciba más fácilmente cuando aparece en el input, y la percepción es considerada por muchos investigadores como un paso indispensable para la adquisición como conocimiento implícito. Pero, tal y como se ha comentado al principio de esta sección, la parte de conocimiento explícito que se transforma en implícito es ínfimo, ya que solo una pequeña porción de propiedades gramaticales de una lengua pasa a ser aprendida conscientemente, $\mathrm{y}$, por otro lado, el conocimiento explícito solo puede transformarse en implícito si los estudiantes están preparados evolutivamente para la adquisición de cada estructura (Gómez del Estal, 2004: 13). Ya Baralo (1995: 65) había señalado que para que esto suceda es necesario la práctica comunicativa, la interacción y la negociación. A continuación se presenta un esquema (Esquema 2. Adquisición y aprendizaje) en el que se refleja cuál sería el camino trazado por el proceso de aprendizaje y cuál la adquisición. En este esquema se evidencia cómo en la adquisición el hablante ha tenido que superar una serie de pasos previos (atención, análisis, reestructuración, monitorización y verificación) para ser capaz de producir, mientras que en el aprendizaje ni se presta atención ni se analiza el intake, hecho que implicará un conocimiento defectuoso de la lengua y un uso limitado de las formas lingüísticas correctas.

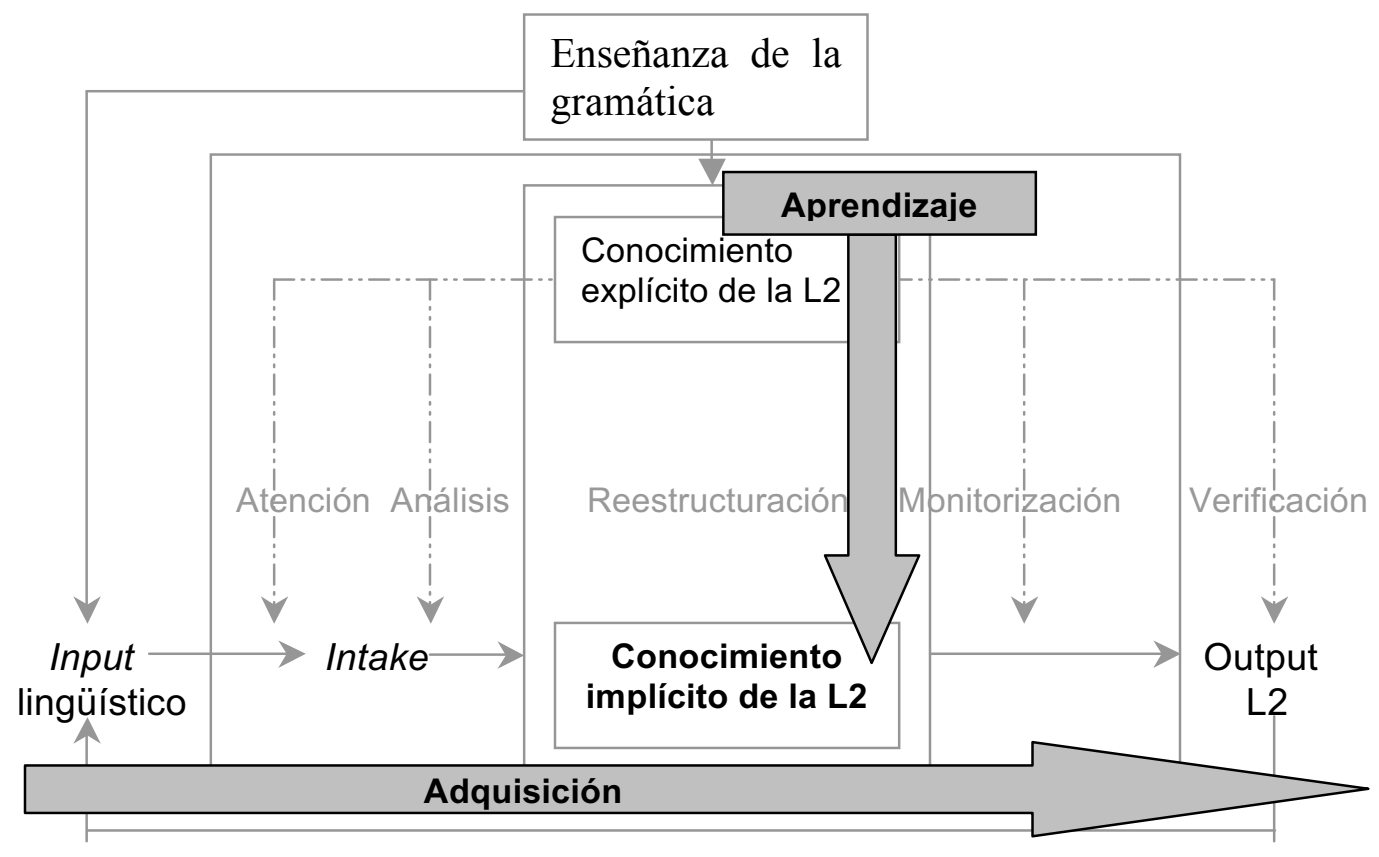

Esquema 2. Adquisición y aprendizaje 
Por último, el conocimiento explícito de las reglas gramaticales desempeña un papel de monitorización cuando se usa la lengua con fines comunicativos (Coronado González, 1998: 82) y acelera la adquisición del conocimiento implícito, pero no es suficiente para que se produzca el intake, ya que es posible que el alumno no esté todavía preparado para asimilar este conocimiento, y por lo tanto no será capaz de producir, el output. Así pues, desde un punto de vista teórico los resultados que los estudiantes obtienen mediante el proceso de adquisición de la lengua -mediante una perspectiva comunicativa y poniendo el foco en la forma- son mejores que aquellos resultados procedentes del aprendizaje. Esta reflexión, será tenida en cuenta a la hora de poner en práctica el material didáctico y establecer unas conclusiones de trabajo.

\section{OBJETIVO DEL ESTUDIO DE CAMPO}

El objetivo general del estudio de campo es evaluar cómo se integra la gramática, puesto que como se ha apuntado en el apartado anterior, existen opiniones diversas sobre cómo afrontar la gramática en el aula de E/LE, y especialmente, cómo integrar la gramática en el ECMT:

No existe un acuerdo sobre el papel de la enseñanza de la gramática, lo cual está directamente relacionado con el debate sobre adquisición y aprendizaje, y sobre cómo este puede influir positivamente (Coronado González, 1998: 82).

Para poder alcanzar este objetivo final sobre la evaluación del componente gramatical en el enfoque comunicativo mediante tareas, se han establecido los siguientes objetivos específicos previos, que permitirán establecer el papel de la gramática en un ECMT:

- Diseñar materiales didácticos - para dos grupos de estudiantes diferentes- con los mismos contenidos, objetivos y tareas finales, pero en el que la enseñanza de la gramática esté claramente diferenciada, para poder evaluar la metodología empleada.

- Establecer el sistema de evaluación del componente gramatical para evaluar cuantitativamente la adquisición (y el aprendizaje) de los alumnos, según el enfoque utilizado en cada grupo.

- Contrastar los resultados de la evaluación mediante dos métodos de enseñanza, para observar la rentabilidad de cada método.

- Destacar resultados más representativos del estudio contrastivo, y proponer, fruto del experimento realizado, unas pautas y recomendaciones en el empleo de una metodología eficaz para la enseñanza y aprendizaje de la gramática en el ECMT. 


\section{Metodología del ESTUdio DE CAMPO}

La principal aportación del presente trabajo quiere ser una evaluación real de los resultados de enseñanza/aprendizaje de la gramática, de acuerdo con las dos metodologías posibles en didáctica: desde una visión explícita y deductiva. Para ello se ha llevado a cabo un experimento de campo sobre dos grupos homogéneos de estudiantes procedentes de programas de intercambio lingüístico en inmersión, y cuya lengua materna no es el español. El experimento se ha realizado en una asignatura impartida en la Universitat de València, lo cual ha permitido garantizar la homogeneidad de los informantes, así como legitimar la presente investigación, gracias a que se ha buscado un número representativo de muestras. Todo de forma crítica, reflexiva y realista.

La asignatura sobre la que se ha realizado el experimento pertenece a los planes de estudios de Graduado europeo en Dirección de empresas (GEDE) 2003 y Graduado en Economía Europea (GEE) 2003, 15667 Español económico y empresarial, situación idónea para llevar a cabo la investigación contrastiva. El objetivo de la asignatura es conseguir que los alumnos sean capaces de desenvolverse en español en el campo de los negocios y de conocer cómo crear una empresa atendiendo las necesidades de la legalidad española y teniendo en cuenta las diferencias socioculturales. A partir del resultado de una prueba de nivel realizada al principio de curso, se han establecido los contenidos lingüísticos (gramaticales y léxicos), a los que se han ido sumando nuevos aspectos de acuerdo con las necesidades observadas en la evaluación continua (tareas entregadas al final de cada una de las unidades y pruebas realizadas cada dos temas, de los seis que componían el curso).

Para que los alumnos lograran el objetivo del curso resultaba necesario ampliar su conocimiento. La materia propuesta en el sílabo estaba estrechamente ligada a la didáctica de E/LE con fines específicos, lo cual ha facilitado el diseño de unidades didáctica mediante el ECMT. Se ha seguido el ECMT para elaborar el material del curso, por lo que se ha establecido una tarea final por cada unidad. Todos los contenidos tratados en cada unidad (contenido funcional, léxico, gramatical y sociocultural) se han diseñado especialmente para que nuestros estudiantes puedan alcanzar el objetivo final de cada unidad, que a su vez, están ligados con la tarea final de curso (la creación de una franquicia). Así pues, el alumno es consciente de que todo el esfuerzo se revertirá en resultados palpables a corto / medio plazo, lo cual favorece su motivación, puesto que el propio aprendiz es conocedor de su progreso. Los diferentes contenidos y las destrezas lingüísticas (comprensión oral, comprensión escrita, expresión oral y expresión escrita) han sido integrados en las unidades didácticas siguiendo las recomendaciones de Estaire y Zanón (1994).

Con la finalidad de constatar la progresión cuantitativa del aprendizaje del alumnado, se han diseñado varias herramientas de diagnóstico y evaluación: una prueba de nivel inicial, tres pruebas de evaluación continua (una de ellas cada dos unidades didácticas) y una prueba final de la asignatura. Asimismo, se han obtenido datos cualitativos gracias a la realización de tareas finales de cada una de las unidades: 
unidad 1: escribir un artículo periodístico sobre economía; unidad 2: realizar un diálogo en un banco; unidad 3: diseñar un cartel con las normas de seguridad de la empresa; unidad 4: establecer el marketing relacional y el marketing de masas de la franquicia que iba a crear, y la elaboración de una tarea final de curso consistente en la creación de una franquicia; unidad 5, realización de un informe sobre la creación de una franquicia; unidad 6: defensa oral.

El material derivado de las actividades realizadas durante el curso, tanto escritas como orales han sido digitalizadas para facilitar su posterior análisis. También se han digitalizado todas las tareas finales corregidas, la prueba de nivel, los test de evaluación continua, el examen final y la tarea final de curso. Se ha grabado en vídeo la tarea final de la segunda unidad, que consistía en representar un diálogo en un banco, y la exposición de la constitución de una franquicia, que correspondía a la tarea final de curso.

A partir de la información cuantitativa recogida durante el proceso de evaluación se han llevado a cabo estadísticas y gráficas de cada una de las pruebas que muestran empíricamente qué tipo de enfoque gramatical logra mejores resultados en el aula.

\section{RESULTADOS DEL ESTUDIO DE CAMPO Y CONCLUSIONES}

\subsection{Resultados cuantitativos}

A lo largo del estudio de campo se han ido evaluando cuantitativamente el aprendizaje y la adquisición del estudiantado; ha sidomuy importante recoger información cuantitativa y poder conocer en cada momento si los alumnos han superado los objetivos planteados al principio del curso, y los programados a lo largo del semestre.

Se han evaluado por separado el componente gramatical y el componente léxico, que constituyen los pilares del curso de español para fines específicos: Se presentan a continuación los resultados cuantitativos de ambos componentes para poder contrastarlos. Si bien para la presente investigación interesan los contenidos gramaticales, se ofrecen también los resultados obtenidos en la evaluación del vocabulario puesto que pueden servir como punto de referencia para juzgar la evolución en ambos grupos y para ver si el camino de aprendizaje ha ido a la par en los dos componentes.

Durante el semestre, cada dos unidades, se han realizado pruebas de evaluación para efectuar un seguimiento exhaustivo de la evolución del aprendizaje. Estos datos nos servían para conocer las carencias de los alumnos, y favorecer el aprendizaje, y poder revisar, llegado el momento, estos aspectos. En las unidades 1 y 2 también se han realizado estas pruebas, pero no se han considerado en nuestro análisis ya que el enfoque con el que trabajábamos la gramática era el mismo, porque se estaba realizando un acercamiento de la metodología a los estudiantes, y queríamos que conocieran el sistema de aprendizaje en la asignatura, antes de poner en marcha la investigación sobre la enseñanza de la gramática. 
Las pruebas se debían efectuar cada dos unidades, y el examen final de curso se realizaba al final del semestre. Las pruebas tanto del grupo de Gramática Explícita (GE) como del grupo de Gramática Implícita (GI) eran iguales. En cada una de las pruebas se disponía de un mismo número de actividades que respondía a la metodología empleada en GE y a la GI, de esta manera, gracias al perfil de examen homogéneo los datos arrojados pueden ser comparados y analizados.

Un último aspecto que se debe tener en cuenta en la evaluación, es la realización de una tarea final de curso, en la que se recogen todos los conocimientos estudiados a lo largo del curso. Se trata de la memoria del plan de negocio para abrir una franquicia, si se ha seguido todo el curso, el estudiante no tiene ningún problema para elaborar esta memoria. Este trabajo debe ser realizado en parejas, y finalmente defendido de forma oral ante el profesor, que puede formular preguntas si encuentra algún detalle que deba destacar.

Veamos los resultados obtenidos en las diferentes pruebas. Gracias a la prueba de nivel, que se realizó el primer día, se pueden ver reflejados los conocimientos previos de los estudiantes. La prueba de nivel tenía el objetivo de conocer las necesidades reales un grupo de estudiantes en concreto $\mathrm{y}$, de esta forma, poner más énfasis en unos aspectos gramaticales o de vocabulario, o en otros.

\begin{tabular}{|l|c|c|} 
& $\begin{array}{c}\text { Grupo 1 } \\
\text { Gram. explícita }\end{array}$ & $\begin{array}{c}\text { Grupo 2 } \\
\text { Gram. implícita }\end{array}$ \\
\hline Gramática & $52,1 \%$ & $48,6 \%$ \\
\hline Vocabulario & $48,6 \%$ & $45,4 \%$ \\
\hline
\end{tabular}

Tabla 2. Resultados de la prueba de nivel

En las unidad 3 y en la unidad 4 no se aprecia una diferencia muy significativa en cuanto a la puntuación entre los dos grupos. Pero, si se tiene en cuenta que tanto en la prueba de nivel como en la prueba de las unidades 1 y 2, el grupo 2 tenía menos puntuación, el resultado de esta prueba es un índice de mejora.

\begin{tabular}{|l|c|c|} 
& $\begin{array}{c}\text { Grupo 1 } \\
\text { Gram. explícita }\end{array}$ & $\begin{array}{c}\text { Grupo 2 } \\
\text { Gram. implícita }\end{array}$ \\
\hline Gramática & $76,4 \%$ & $77 \%$ \\
\hline Vocabulario & $78,4 \%$ & $77,9 \%$ \\
\hline
\end{tabular}

Tabla 3. Resultado de la prueba de las unidades 3 y 4

Sin embargo, sí que podemos apreciar una elevadísima diferencia en las unidades 5 y 6 , en donde el grupo que ha adquirido la gramática de forma implícita supera en más de un $12 \%$ la puntuación obtenida por el otro grupo. 


\begin{tabular}{|l|c|c|} 
& $\begin{array}{c}\text { Grupo 1 } \\
\text { Gram. explícita }\end{array}$ & $\begin{array}{c}\text { Grupo 2 } \\
\text { Gram. implícita }\end{array}$ \\
\hline Gramática & $58,60 \%$ & $71 \%$ \\
\hline Vocabulario & $65,7 \%$ & $69,5 \%$ \\
\hline
\end{tabular}

Tabla 4. Resultado de la prueba de las unidades 5 y 6

El examen final de curso tenía dos partes. En la primera se presentaban preguntas de respuesta breve, completar huecos y reescritura, y una segunda parte que era de tipo test.

\begin{tabular}{|l|c|c|}
\multicolumn{1}{c|}{} & $\begin{array}{c}\text { Grupo 1 } \\
\text { Gram. explícita }\end{array}$ & $\begin{array}{c}\text { Grupo 2 } \\
\text { Gram. implícita }\end{array}$ \\
\hline Gramática & $68,7 \%$ & $75,3 \%$ \\
\hline Vocabulario & $87,2 \%$ & $85,2 \%$ \\
\hline
\end{tabular}

Tabla 5. Resultado de la prueba de la prueba final, parte escrita

Se puede observar como la puntuación obtenida en el apartado de gramática por el grupo de GI es superior a la conseguida por el GE, no obstante, si se atiende a la puntuación en el apartado de vocabulario -trabajado en ambos grupos de la misma forma- se observa que la puntuación obtenida es ligeramente superior en el primero de los grupos. En la prueba tipo test también se obtienen las mismas diferencias.

\begin{tabular}{|l|c|c|} 
& $\begin{array}{c}\text { Grupo 1 } \\
\text { Gram. explícita }\end{array}$ & $\begin{array}{c}\text { Grupo 2 } \\
\text { Gram. implícita }\end{array}$ \\
\hline Gramática & $64,6 \%$ & $68,3 \%$ \\
\hline Vocabulario & $75,8 \%$ & $70,6 \%$ \\
\hline
\end{tabular}

Tabla 6. Resultado de la prueba de la prueba final, tipo test

De nuevo el grupo 2, GI, supera la puntuación del GE. Y es bastante significativo que en el vocabulario, el grupo GI, obtenga menor puntuación. Las diferencias observadas en los resultados de los alumnos en las diversas pruebas ofrecen evidencias de que tanto los alumnos que trabajan la gramática de forma explícita como los que lo hacen de forma implícita consiguen avanzar en su aprendizaje; sin embargo, se aprecia cómo en el caso de los alumnos de GI, su aprendizaje es más eficaz.

Si recogemos en una misma gráfica las puntuaciones en gramática a lo largo de todo el curso, observamos que los estudiantes de gramática implícita han obtenido constantemente mejores resultados, a pesar de que en la prueba realizada el primer día obtuvieron resultados más bajos, y por lo tanto el punto de partida fue inverso. 


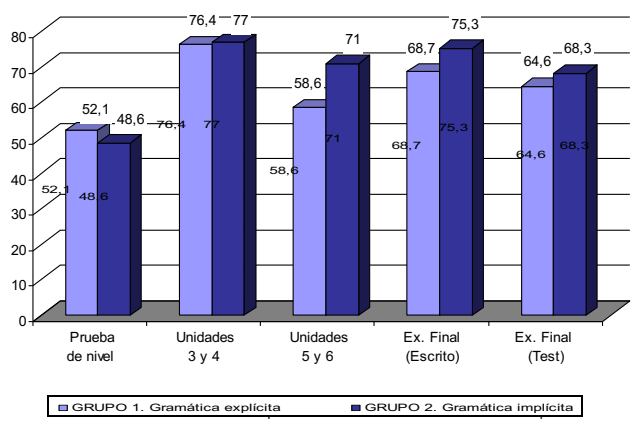

Gráfica 1. Puntuación obtenida en el apartado de gramática en las pruebas y los exámenes (porcentaje)

\begin{tabular}{|l|c|c|} 
& $\begin{array}{c}\text { Grupo 1 } \\
\text { Gram. explícita }\end{array}$ & $\begin{array}{c}\text { Grupo 2 } \\
\text { Gram. implícita }\end{array}$ \\
\hline Prueba de nivel & $52,1 \%$ & $48,6 \%$ \\
\hline Unidades 3 y 4 & $76,4 \%$ & $77,0 \%$ \\
\hline Unidades 5 y 6 & $58,6 \%$ & $71,0 \%$ \\
\hline Ex. final-escrito & $68,7 \%$ & $75,3 \%$ \\
\hline Ex. final-test & $64,6 \%$ & $68,3 \%$ \\
\hline
\end{tabular}

Tabla 7. Puntuación obtenida en el apartado de gramática en las pruebas y los exámenes

Por lo tanto, podemos concluir que el grupo que ha adquirido la gramática de forma implícita ha asimilado mejor los contenidos (Martí Contreras, 2014).

\subsection{Conclusiones}

Ha quedado demostrado, por los datos que se han extraído de los exámenes, de las evaluaciones, de las tareas entregadas al final de cada unidad, del proyecto final de curso, así como a través de las grabaciones, que los alumnos que han trabajado con gramática implícita han sido capaces de adquirir mejor los conocimientos gramaticales que los alumnos que estudiaban con la gramática explícita

Aunque el tiempo que se dedica a cada aspecto gramatical es importante para favorecer el aprendizaje, nuestros resultados muestra que el éxito en el aprendizaje no reside tanto en el tiempo dedicado a desarrollar y asimilar cada fenómeno gramatical, como en el modo de trabajarla para adquirirlo. En nuestro caso, se ha dedicado aproximadamente el mismo tiempo en total a cada uno de los aspectos gramaticales en ambos grupos del estudio de campo. Si se contextualiza la gramática, sobre todo con documentos reales, vinculados con el área de estudios de los alumnos con los que se ha experimentado (negocios y economía), el resultado final del aprendizaje es mucho más satisfactorio que si se abordan elementos que no están vinculados entre sí.

También se observa cómo hay una tendencia ano trabajar de forma individual, sino que, por la misma naturaleza de las actividades -incluso para realizar tareas en las que aparece la gramática-, es mucho mejor la agrupación en parejas o pequeños grupos para favorecer las destrezas lingüísticas y las habilidades comunicativas. 
El rol del profesor en clase es totalmente diferente al rol del profesor en la enseñanza tradicional, por dos motivos. El primero de ellos afecta a los dos grupos. Los materiales que se han diseñado para el curso siguen un enfoque comunicativo mediante tareas, por lo que todas las actividades realizadas en cada unidad, han estado dirigidas a la resolución de una tarea y a un proyecto final de curso. Tal como queda reflejado en los datos, el profesor tiene el papel de guiar el aprendizaje. El alumno, y no el profesor, constituye, el centro de aprendizaje. En este sentido, se ha facilitado a los estudiantes semanalmente los materiales para el seguimiento de la asignatura. Por el contrario, no han dispuesto de un manual didáctico que debían seguir desde el principio de curso: se pretendía que lo visto en clase estuviera adaptado a las necesidades específicas de los alumnos de esta asignatura. El segundo de los motivos es el enfoque explícito o implícito empleado en los grupos. Según aparece en los datos recogidos se observa en GI que el profesor apenas explica aspectos gramaticales de forma directa, sino que logra que reflexionen sobre las normas sin necesidad de explicitarlas (solo 40 minutos durante todo el tiempo analizado, mientras que en GE son necesarias más de dos horas en explicaciones gramaticales).

Por último, el papel del estudiante es lo más importante en el proceso de aprendizaje, ya que en torno a él gira toda la enseñanza. A principio de curso realizaron una prueba de nivel para evaluar los conocimientos previos, y a partir de los resultados obtenidos, se establecieron los objetivos que se debían cumplir y las necesidades específicas que se pretendía abordar. El resultado de este modo de operar, unido al desarrollo de los métodos de aproximación a la gramática, ha mostrado resultados muy elocuentes. Hemos observado que desde una aproximación a la gramática implícita, los alumnos son quienes descubren las normas gramaticales, gracias a las actividades planteadas por el profesor. La deducción / inducción de las normas gramaticales implica una reflexión que lleva a una adquisición de la gramática, no solo a un aprendizaje. De la dicotomía adquisición (GI) versus aprendizaje (GE) se muestra -y se demuestra- a partir de los resultados del presente estudio empírico, que si se tiene que puntuar matemáticamente la adquisición-aprendizaje, los alumnos que han estudiado con el método GI obtienen mejores resultados.

Además, no solo es mejor el resultado de la calificación del alumnado en conocimientos gramaticales, sino que también la metodología utilizada a lo largo de todo el curso ha favorecido un ambiente participativo en el aula, así como la implicación de los estudiantes en el proceso de aprendizaje-enseñanza, tal y como se demuestra en los datos arrojados por las estadísticas de la asignatura, del desarrollo del curso y del profesor, facilitadas por la Unitat de qualitat de la Universitat de València.

\section{REFERENCIAS BIBLIOGRÁFICAS}

Aguado-OreA, Javier y Marta BARALO (2007): «Aspectos teóricos y metodológicos de la investigación sobre el aprendizaje léxico y gramatical del español como L2», Revista de Educación, vol. 343, 113-32. 
Alonso Raya, Rosario (2010): «Cómo mejorar tu vida con la gramática», Revista de Didáctica MarcoELE, monográficos, vol. 10, 1-12.

Alonso Raya, Rosario y Pablo Martínez Gila (1997): «Prácticas gramaticales. Criterios de presentación y secuenciación», en Jiménez, T., M. C. Losada y J. F. Márquez, eds., Español como Lengua Extranjera: Enfoque comunicativo y gramática IX Congreso Nacional ASELE, Santiago de Compostela, ASELE, 325-331.

AMBJOERN, Lone (2009): «Desarrollo del conocimiento explícito gramatical basado en el procesamiento del input: una perspectiva inductiva», RedELE, Revista electrónica de didáctica de Español como Lengua Extranjera, vol. 17, s/p.

BARAlo OtTONELlo, Marta (1995): «Adquisición y/o aprendizaje del español/LE» en Rued, M. et al. (eds.) Tendencias Actuales en el Español como Lengua Extranjera II: VI Congreso Nacional ASELE, León, ASELE, 63-68.

BARAlo OtTONEllo, Marta (1996): «La teoría lingüística y la teoría de la adquisición de lengua extranjeras», REALE: Revista de estudios de adquisicón de la lengua española, vol. 5, 9-42.

Baralo OtTonello, Marta (2009): «Reflexiones sobre la adquisición de la gramática y su implicación en el aula», Revista de Didáctica MarcoELE, 7-18.

BARDOVI-HARLIG, Kathleen (2003): «Understanding the role of grammar in the acquisition of L2 Pragmatics»), en Martínez Flor, Alicia et al., eds., Pragmatic Competence and Foreign Language Teaching, Castelló, Publicacions de la Universitat Jaume I, 25-44.

BRUCART, José María (1997): «Gramática y adquisición en la enseñanza del español como lengua extranjera», en Celis, Á. y J. R. Heredia, eds., Lengua y Cultura en la Enseñanza del Español a Extranjeros: VII Congreso Nacional ASELE, Almagro. UCM, ASELE, 63-68.

CASTAÑEdA CASTRO, Alejandro (1988): «Enseñanza comunicativa de la gramática: actividades para el aula», en Fente Gómez, R., A. Martínez González y J. A. Molina Redondo, eds., El Español como Lengua Extranjera: Aspectos Generales: I Congreso Nacional ASELE, Granada, 67-78.

CONSEJO DE EUROPA (2002): Marco común europeo de referencia para las lenguas: aprendizaje, enseñanza, evaluación Madrid: Ministerio de Educación, Cultura y Deporte, Instituto Cervantes/ Anaya, 2002 [en línea]: $<$ http://cvc.cervantes.es/ensenanza/ biblioteca ele/marco/>. [Consulta: 5/4/15].

CORONADO GONZÁLEZ, M. ${ }^{a}$ Luisa (1998): «Ultimas aportaciones a la enseñanza de la gramática: aproximación inductiva, actividades de concienciación gramatical y tareas formales», Carabela, vol. 43, 81-94.

ELLIS, Rod (2003 [1994]): The Study of Second Language Adquisition, Oxford, Oxford University Press.

ESTAIRE, Sheila y Javier ZANÓN (1990): «El diseño de unidades didácticas en L2 mediante tareas: principios y desarrollo», Comunicación, Lenguaje y Educación, vol. 7/8, 55-90, < http://dx.doi.org/10.1080/02147033.1990.10820942>.

FUENTES DE LA ROSA, Carmen Ceular (1993): «El aprendizaje y la enseñanza de las lenguas modernas», en García Hoz, V., ed., Enseñanza y aprendizaje de lenguas modernas, Madrid, RIALP, 109-158. 
GÓMEZ Del Estal VillaRinO, Mario (2004): «La enseñanza de la gramática dentro del enfoque por tareas», en Gómez Asencio, J. y J. Sánchez Lobato, Forma 8: gramática, enseñanza y análisis, Madrid, SGEL, 83-107 [en línea]: $<$ http://cvc.cervantes.es/ensenanza/

biblioteca ele/antologia didactica/gramatica/gomez del estal01.htm>. [Consulta: 5/6/14].

Gómez del Estal Villarino, Mario y Javier ZANÓn GóMEZ (1996): «La enseñanza de la gramática mediante tareas», en MonTESA, S. y S. GoMIS, eds., Tendencias Actuales en el Español como Lengua Extranjera: V Congreso Nacional ASELE. Málaga, ASELE, 89-100.

GUTIÉRREZ ARAUS, María Luz (1998): «La instrucción gramatical en el enfoque comunicativo», en Jiménez, T., M. C. Losada y J. F. Márquez, eds., Español como Lengua Extranjera: Enfoque comunicativo y gramática IX Congreso Nacional ASELE, Santiago de Compostela. Santiago de Compostela, ASELE, 111-116

IZQUIERDO, Carmen (2008): La gramática en los manuales de español para fines específicos. Memoria final de Máster de Profesores E/LE. Universidad de Barcelona Virtual. Curso 2006/2008.

LARSEN-FrEEMAN, Diane y Michael H. LONG (1994 [1991]): Introducción al estudio de la adquisición de segundas lenguas, Madrid, Gredos.

LEECH, Geoffrey N. (1989): "Grammar and Language Learning», Actas del VII Congreso Nacional de AESLA, Sevilla, Universidad de Sevilla, 15-25.

LlOPIS GARCÍA, Reyes (2009): «La instrucción gramatical en la Adquisición de Segundas Lenguas: revisión de ayer para propuestas de hoy», RedELE, vol. 16, 1-15.

Llopis García, Reyes, Juan Manuel ReAL EsPINOSA y José Domingo RUIZ CAMPILlO (2010): Qué gramática enseñar, qué gramática aprender, Madrid, Edinumen.

MATTE Bon, Francisco (1998): «Gramática, pragmática y enseñanza comunicativa del español como lengua extranjera», Carabela, vol. 43, 53-79.

MARTí CONTRERAS, Jorge (2014): Evaluación del componente gramatical en el enfoque comunicativo por tareas en la enseñanza de Español como Lengua Extranjera, tesis doctoral, Valencia, Universitat de València [en línea]: $<$ http://roderic.uv.es/handle/10550/36294 $>$. [Consulta: 16/3/15].

ORTEGA Olivares, Jenaro (1998): «La instrucción gramatical en el enfoque comunicativo», en Jiménez, T., M. C. Losada y J. F. Márquez, eds., Español como Lengua Extranjera: Enfoque comunicativo y gramática IX Congreso Nacional ASELE, Santiago de Compostela: ASELE, 133-142.

Rodríguez Abella, Rosa María y M. VAlero Gisbert (1998): «La gramática para comunicar: una propuesta inductiva», en Jiménez, T., M. C. Losada y J. F. Márquez, eds., Español como Lengua Extranjera: Enfoque comunicativo y gramática IX Congreso Nacional ASELE, Santiago de Compostela, ASELE, 433-440.

RuIZ CAMPILLO, José Plácido (2007): «Gramática cognitiva y E/LE, entrevista a José Plácido Ruiz Campillo», Revista de Didáctica MarcoELE, vol. 5, 1-17.

SALAZAR, Ventura (2006): "Gramática y enseñanza comunicativa del españollengua extranjera», Revista de Didáctica MarcoELE, vol. 2, 1-34. 
SÁnchez PÉrez, Aquilino (2000 [1997]): Los métodos en la enseñanza de idiomas. Evolución histórica y análisis didáctico, Alcobendas, SGEL.

VanPatten, Bill (1996): Input processing and Grammar Instruction in Second Language Acquisition, Norwood, Ablex.

WIDODO, Handoyo (2006): «Approaches and procedures for teaching grammar», en English Teaching: Practice and Critique, Waikato, University of Waikato, 122-141 [en línea]: <https://education.waikato.ac.nz/ research/files/etpc/2006v5n1nar1.pdf $>$. [Consulta: 16/3/15].

VIÑA RouCO, Mar (2005): «Metodología inductiva y deductiva en la enseñanza de las lenguas vivas en España en el siglo XIX», Porta Linguarum, vol. 4, 185-200.

WINTER, B. y A. S. REBER (2003 [1994]): «Implicit learning and natural language acquisition», en Ellis, Rod, The Study of Second Language Adquisition, Oxford, Oxford University Press.

ZANÓN GóMEZ, Javier y María José HeRnÁNDEZ (1990): «La enseñanza de la comunicación en clase de español», Cable, vol. 5, 12-18.

\section{ABREVIATURAS UTILIZADAS}

E/LE Español como Lengua Extranjera

ECMT Enfoque comunicativo mediante tareas

L1 Lengua materna

L2 Segunda lengua

MCER Marco común europeo de referencia para las lenguas 